\title{
Enhancement of Accuracy of Hand Shape Recognition Using Color Calibration by Clustering Scheme and Majority Voting Method
}

\author{
Takahiro Sugaya, Hiromitsu Nishimura, and Hiroshi Tanaka \\ Kanagawa Institute of Technology, \\ 1030 Shimo-ogino, Atsugi-shi, Kanagawa, Japan \\ s1385015@cce. kanagawa-it.ac.jp, \\ \{nisimura,h_tanaka\} @ic.kanagawa-it.ac.jp
}

\begin{abstract}
This paper presents methods of enhancing the recognition accuracy of hand shapes in a scheme which is proposed by the authors as being easy to memorize and which can represent much information. To ensure suitability for practical use, the recognition performance must be maintained even when there are changes in the illumination environment. First, a color calibration process using a k-means clustering scheme is introduced as a way of ensuring high performance in color detection. In the proposed method the thresholds for hue values are decided before the recognition process, as a color calibration scheme. The second method of enhancing accuracy involves making a majority decision. Many image frames are obtained from one hand shape before the transition to the next shape. The frames in this hand shape formation time span are used for shape recognition by majority voting based on the recognition results from each frame. It has been verified by carrying out experiments under different illumination conditions that the proposed technique can raise the recognition performance.
\end{abstract}

Keywords: Color Gloves, Shape Recognition, Color Detection, Hue Value, Clustering, Majority Voting, Illumination Environment.

\section{Introduction}

Fingerspelling and sign language are the largest barriers to independent living for hearing impaired or speech-impaired persons. However, it is difficult to acquire the necessary deciphering skills as well as to learn the skills to express meaning. Gesture recognition systems etc. have been developed in order to solve these problems [1]. The methods which use a special sensor or devices [2, 3] involve a high cost of introduction or the need to attach sensors to the human body. In addition, since some systems have restrictions on the locations where they can be used, due to the illumination conditions, it is difficult to use these systems as universal communication tools for handicapped people. 
We have proposed a single vision-based hand shape recognition method, because it does not require sensors to be attached to the human body. A camera implemented in a smartphone, etc. may be applied to this method. There are two kinds of approach used in methods for vision-based recognition. One is methods using color markers, such as color gloves, and the other is methods which do not use color markers [4]. Of course, the recognition method that does not use special markers is more userfriendly. However, in the research on sign language recognition in which a color glove or markers were not used [5,6], the complicated shape of fingers or their motion could not be accurately detected. On the other hand, although color gloves were used for hand shape recognition in another study [7], a lot of colors were used, and the evaluation test was carried out under only one illumination condition. In order to realize a hand shape recognition system which can identify each fingertip in a simpler and more robust manner, we decided to use a color glove in which a different color was assigned to each fingertip.

In general, the use of color detection techniques alone cannot maintain high detection performance in an environment of varying light conditions. Color detection is one of the most important elements in shape recognition. In our previous study [8], it was proposed to achieve high sensitivity color detection by considering thresholds for determining the hue values under different illumination conditions. However, shape recognition errors cannot be eliminated because of color detection errors which still occur in some illumination environments. It was clarified that the shape recognition performance must be improved in order to achieve satisfactory practical use.

This paper presents a means of enhancing the accuracy of hand shape recognition. First, a color calibration process using a k-means clustering scheme is introduced for color detection regardless of any difference of illumination conditions. Second, the final hand shape recognition results are determined by majority voting based on the recognition results from many image frames which are extracted from a single hand shape formation period. It is verified by experiments carried out under various illumination conditions that our proposed hand shape recognition system using these two methods can be achieved high recognition results.

\section{Recognition Target and Color Detection}

The authors have proposed the use of color gloves on each hand, with the fingers marked with different colors, for hand shape recognition. Color gloves have an advantage in facilitating the recognition of hand shapes in comparison with recognition by contour abstraction of hand shapes, especially when taking into account the background environment and the need for discrimination between fingers. Six colors were selected by considering their hue values, since these are used to discriminate between the colors. The portions of the glove that are colored are the tip of each finger and the wrist as shown in Fig.1. We propose a new set of finger patterns which is easy to memorize. Each finger represents a binary number, where 1 means visible from the camera, and 0 means an invisible finger, that is a finger which 
is folded back and not extended. The order of digits in a number follows the order of fingers, that is, the thumb represents the LSB (right-most position of 5 bits), and the little finger corresponds to the MSB, for both right and left hands. We propose that the viewing region of single camera is divided into two separate regions, namely, right hand and left hand regions. If the crossing of hands during use is not allowed, the two regions are independent and a large number of signals can be easily created.

The recognition process is performed using the scheme of "nearest neighbor", in which the result is selected on the basis of the shortest distance between template vectors of defined shapes and the feature vector of hand shape $\mathbf{f}$, shown in Fig.1, provided by the camera image. The elements are the distances from the center of the wrist to each colored finger tip region, where element value 0 is used to indicate a colored region which is invisible due to a finger being bent. The shape recognition result is obtained by selecting the hand shape which has the minimum distance between template feature vectors prepared in advance and the target feature vector which is to be recognized.

The hand shape recognition performance is governed by the color detection characteristics. Because the hand shape is recognized on the basis of the positions of each finger tip, these positions are defined as the center of gravity of each colored region. The color detection is based on the hue values for each color. These values are influenced by the illumination environment, that is, brightness and light direction. Color detection errors include the following three cases, that is, (i) color is detected in a different position to the one it should be in, (ii) color cannot be detected in the position where it should be, (iii) color is detected in a position that it should not be in (over detection).

First, the authors investigated highly accurate color detection to achieve high recognition performance. The characteristics of the color detection are strongly influenced by the illumination environment, because the hue value for each colored area depends on the illumination conditions.

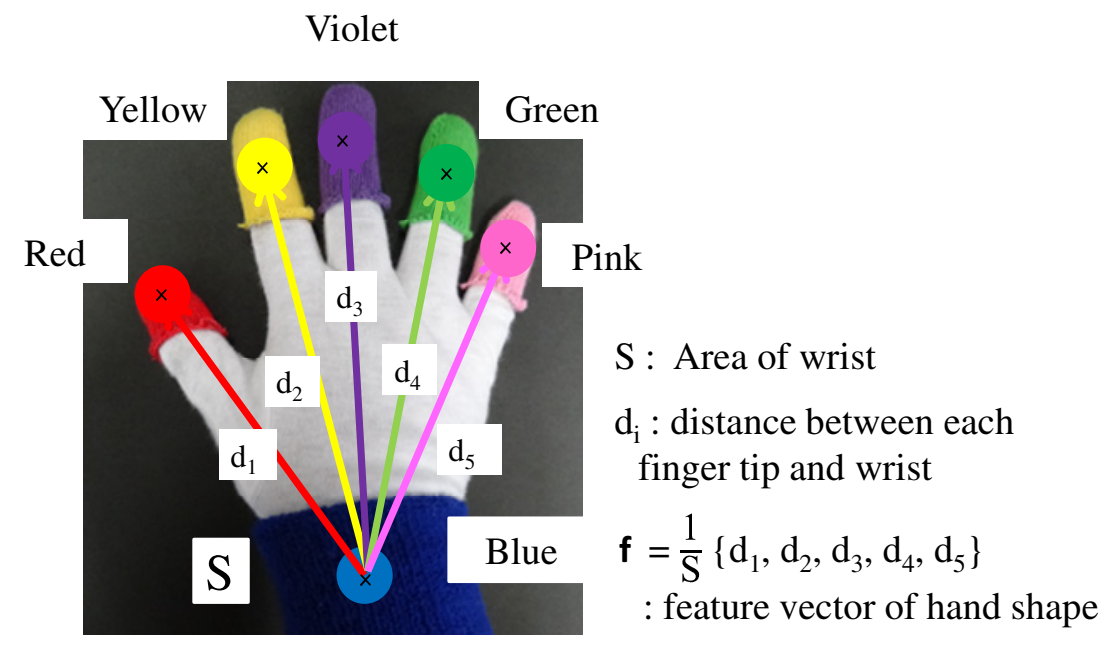

Fig. 1. Color glove for shape recognition 


\section{Color Threshold Calibration for Color Detection}

\subsection{Hue Value Decision Threshold}

It is necessary to discriminate six colors correctly under any illumination conditions. The authors propose calibration of the color thresholds before the shape recognition process commences, since the hue values are altered by the illumination conditions. Therefore we propose that these values are determined by a clustering scheme. Although this clustering imposes a computing load, the k-means clustering scheme was applied in this investigation. Since the clustering operation is carried out only once, and before the recognition process, it does not result in any delay in the recognition process itself. In addition, the initial value for clustering can be easily decided by taking the nominal hue values of each color into account. This scheme can be considered as color threshold calibration for color detection, to eliminate the effect of the illumination environment and the particular camera to be used.

The proposed scheme involves the following processing, as shown in Fig.2.

1. Creating of a histogram of hue values from the image of the hand taken by the camera

2. Introduction of preprocessing before clustering from the histogram, that is, the removal of low saturation color (white), suppression of maximum values (limiting) and removal of infrequently occurring hue values as noise

3. Clustering by k-means to determine the center of the hue values for the 6 colors from the histogram data

4. Determination of hue value range for the detection of each color using the clustering result and the hue value range based on past experimental results

In addition, the following treatments are undertaken in k-means processing by taking the features of 6 colors into account.

5. Initial values of hues are decided by hues under nominal illumination conditions

6 . The differences of each center of hue value are more than 3 degrees by considering the color being used.

7. Hue values which had zero appearance in the histogram were ignored.

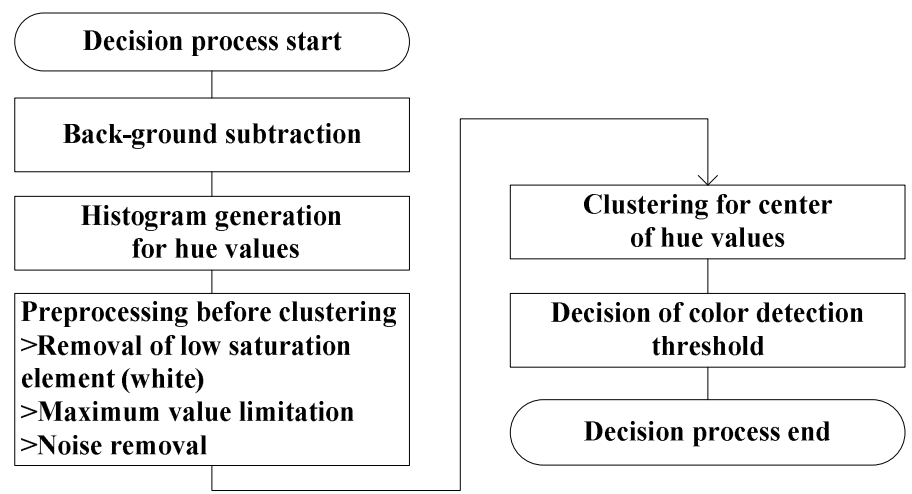

Fig. 2. Proposed hue value threshold decision process 


\subsection{Experimental Conditions and Results}

$\mathrm{k}$-means clustering processing was applied for deciding the center of the hue values of each color. Three different physical locations were selected for comparing the difference of hue values by k-means clustering. The experimental locations were, a place far from a window, a place at mid-distance from the window and a place near to the window, as shown in Fig.3. The experimental conditions such as room light, sun light from the window and background are summarized in Table 1. A Web camera (Logicool HD Pro Webcam C910, 5 million pixels, 30 frames/s) was used in this experiment. The camera was mounted on the notebook PC as shown in Fig.3 and the distance between hand and camera was set to about $50 \mathrm{~cm}$.
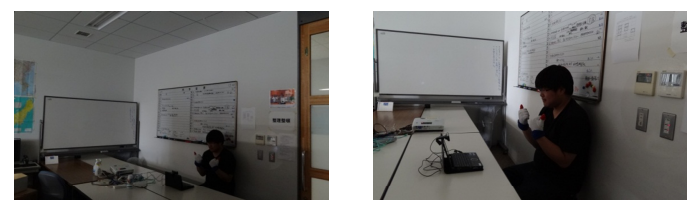

10 Lux
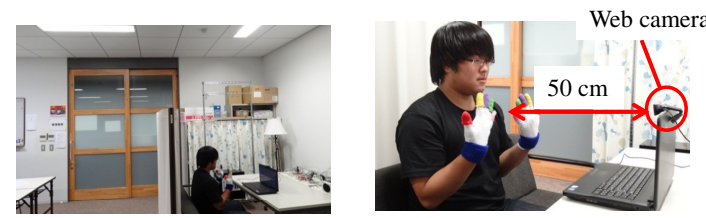

$100 \operatorname{Lux}$
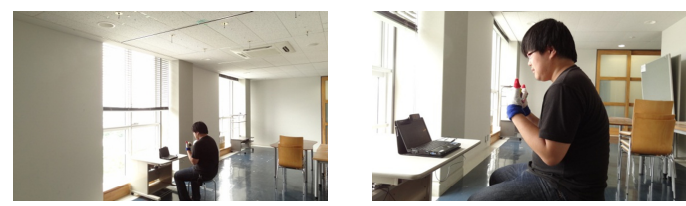

400 Lux

Fig. 3. Experimental environments for color clustering

Table 1. Experimental conditions for color clustering

\begin{tabular}{|c|c|c|c|}
\hline Illumination & Position of room light & Influence of sunlight & Background \\
\hline 10 Lux & $\begin{array}{c}\text { Only overhead light turned on. } \\
\text { All others off }\end{array}$ & Small & White board \\
\hline $100 \mathrm{Lux}$ & Overhead & Small & Wall \\
\hline $400 \mathrm{Lux}$ & All lights turned off & Large & None \\
\hline
\end{tabular}

The hue value ranges for each color obtained is shown in Fig. 4. The centers of gravity of each color were obtained by k-means clustering. The hue value ranges for each color were investigated under several illumination conditions in advance. This method of using color calibration can lead to a decrease in color detection error. 


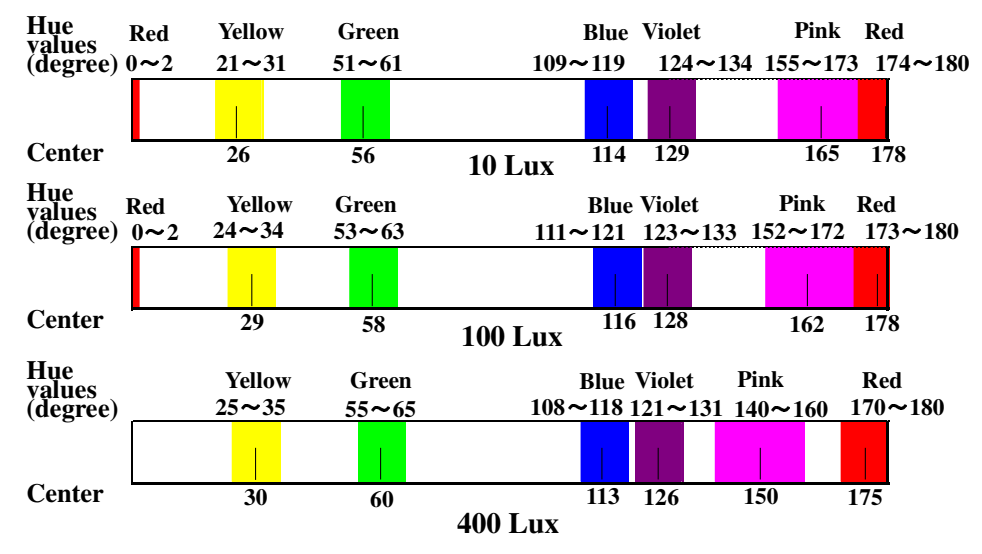

Fig. 4. Example of hue value threshold pattern for color detection

Experiments of the detection of each color of the finger tips were carried out in order to confirm the validity of the proposed system. Table 2 and Table 3 show the experimental results without calibration and with calibration, respectively. In these experiments the cases examined were with "all fingers invisible" and "all fingers visible", and the number of acquired frames was 100 . In the hand shape column, 0 means a folded finger, invisible, and 1 means an extended finger, visible, and the character on the left indicates a thumb, and that on the right a little finger. The success ratios of color detection were confirmed using these hand shapes. It was verified that the color calibration we proposed gives good results for color detection. It was decided to apply this scheme before starting the recognition process, as color calibration to absorb differences in the illumination conditions.

Table 2. Experimental results without color calibration

\begin{tabular}{|c|c|c|c|c|c|c|}
\hline Illumination & $\begin{array}{c}\text { Hand } \\
\text { shape }\end{array}$ & $\begin{array}{c}\text { Total } \\
\text { frames }\end{array}$ & $\begin{array}{c}\text { Success } \\
\text { frames }\end{array}$ & $\begin{array}{c}\text { Error } \\
\text { frames }\end{array}$ & $\begin{array}{c}\text { Detection } \\
\text { success ratio }\end{array}$ & $\begin{array}{c}\text { Average detection } \\
\text { success ratio }\end{array}$ \\
\hline \multirow{2}{*}{10 Lux } & 00000 & 100 & 41 & 59 & $41 \%$ & \multirow{2}{*}{$64.0 \%$} \\
\cline { 2 - 6 } & 1111 & 100 & 87 & 13 & $87 \%$ & \multirow{2}{*}{$85.5 \%$} \\
\hline \multirow{2}{*}{100 Lux } & 00000 & 100 & 71 & 29 & $71 \%$ & \multirow{2}{*}{$88.5 \%$} \\
\cline { 2 - 6 } & 11111 & 100 & 100 & 0 & $100 \%$ & $100 \%$ \\
\hline \multirow{2}{*}{400 Lux } & 00000 & 100 & 100 & 0 & $77 \%$ & \\
\cline { 2 - 5 } & 11111 & 100 & 77 & 23 & & \\
\hline
\end{tabular}

Table 3. Experimental results with color calibration

\begin{tabular}{|c|c|c|c|c|c|c|}
\hline Illumination & $\begin{array}{l}\text { Hand } \\
\text { shape }\end{array}$ & $\begin{array}{c}\text { Total } \\
\text { frames }\end{array}$ & $\begin{array}{l}\text { Success } \\
\text { frames }\end{array}$ & $\begin{array}{l}\text { Error } \\
\text { frames }\end{array}$ & $\begin{array}{c}\text { Detection } \\
\text { success ratio }\end{array}$ & $\begin{array}{c}\text { Average detection } \\
\text { success ratio }\end{array}$ \\
\hline \multirow{2}{*}{$10 \operatorname{Lux}$} & 00000 & 100 & 100 & 0 & $100 \%$ & \multirow{2}{*}{$99.5 \%$} \\
\hline & 11111 & 100 & 99 & 1 & $99 \%$ & \\
\hline \multirow{2}{*}{$100 \mathrm{Lux}$} & 00000 & 100 & 100 & 0 & $100 \%$ & \multirow{2}{*}{$93.5 \%$} \\
\hline & 11111 & 100 & 87 & 13 & $87 \%$ & \\
\hline \multirow{2}{*}{400 Lux } & 00000 & 100 & 100 & 0 & $100 \%$ & \multirow{2}{*}{$99.5 \%$} \\
\hline & 11111 & 100 & 99 & 1 & $99 \%$ & \\
\hline
\end{tabular}




\section{$4 \quad$ Frames Used for Recognition Process and Majority Decisions}

\subsection{Duration of Each Hand Shape Formation}

The hand shape recognition is carried out after color calibration, which involves a clustering process, as described in the previous section. The hand shape to be recognized is a static shape, not moving. We must separate each hand shape in time from the one before and the one after.

We use the hand shape with all fingers folded, namely "rock" (as in paper-rockscissors) to indicate the end of each hand shape. In this shape, no finger color can be found in the camera image, except for the blue painted wrist band. Figure 5 shows an example of movement of the center of gravity of the colored regions on the fingers. Value 0 means no movement, which means the hand shape has returned to the "rock" shape after forming a hand shape. The authors regard the time span from one 0 value, that is, "rock" shape to the next stable 0 value as the hand shape formation span. The shaded areas indicate no color detection. This sometimes occurs for a short period as indicated in Fig.5. We decided to neglect such short periods, so this region is not considered as a "rock" shape indicating the end of a shape formation. Lack of color detection for more than 5 successive frames (frame rate : $30 \mathrm{fps}$ ), namely about $0.17 \mathrm{~s}$, is interpreted as the end of a shape.

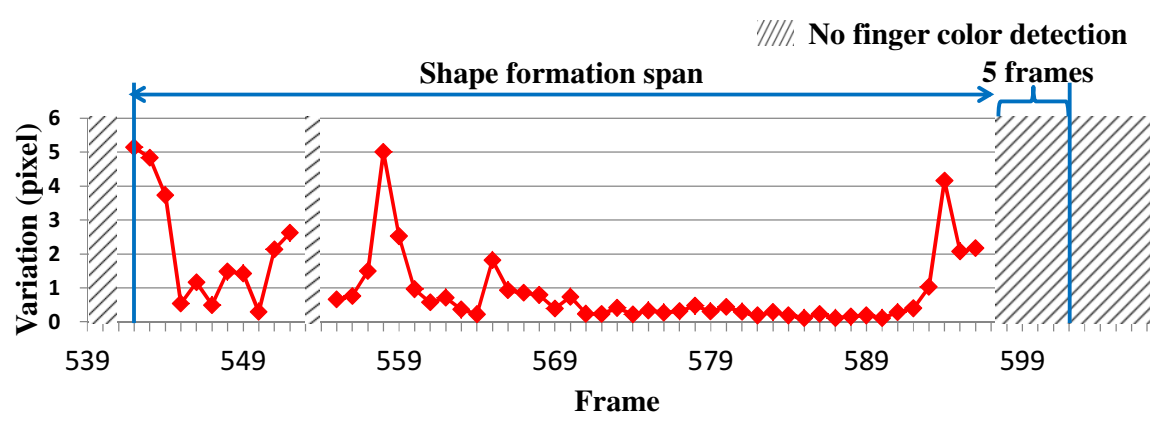

Fig. 5. Movement of center of gravity of colored regions on fingers

\subsection{Shape Recognition Sequence and Majority Decisions}

Figure 6 shows the distance between the target hand shape and templates vectors in the shape formation span. The templates are prepared in advance. The target hand shape vector to be recognized is created by a camera image, and the distance is calculated from each template, each template corresponding to a different hand shape. The number of the templates is 31 in this experiment. The frame rate is about $30 \mathrm{fps}$.

The distance is not constant as shown in this figure due to finger motion. In this example, it is verified that the effect of finger motion and lack of color detection occurred were found in the left section. These errors affect the distances and could lead to recognition error if only one frame were used for the recognition process. 
Therefore we apply a majority decision based on all the recognition results obtained using the frames for recognition, which are the frames in the second half of the shape formation span. This is expected to lead to an enhancement in recognition performance.

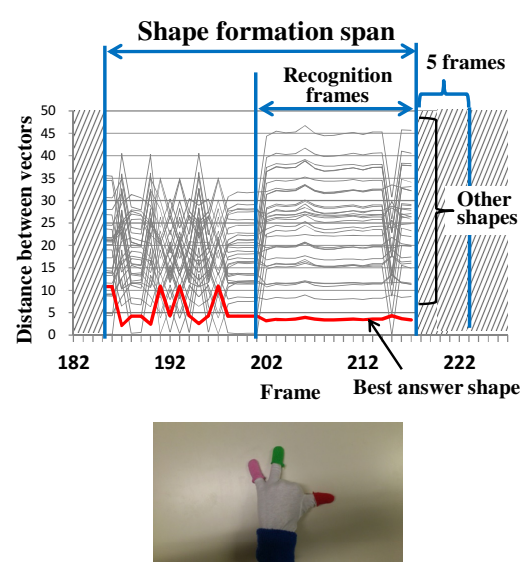

Hand shape (11001)
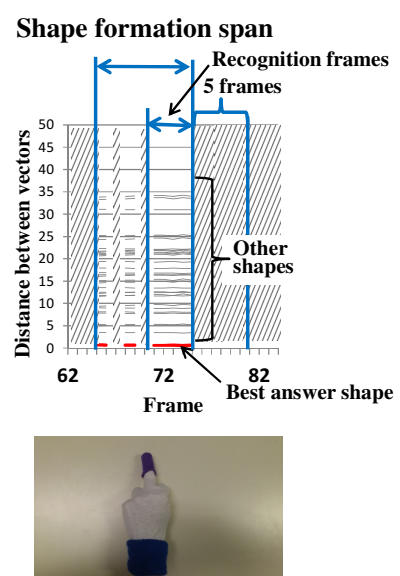

Hand shape (00100)

Fig. 6. Distance between target hand shape vector and template vectors

The shape recognition sequence is summarized in Fig.7. The color calibration is carried out before creating the feature vector. The validity of the feature vector is confirmed by considering the hand shape itself, that is, the relationship of the positions of each finger and the finger lengths. Although the recognition result is output after hand shape formation in this sequence, the delay does not become problem because the image capturing rate and recognition process are sufficiently rapid.

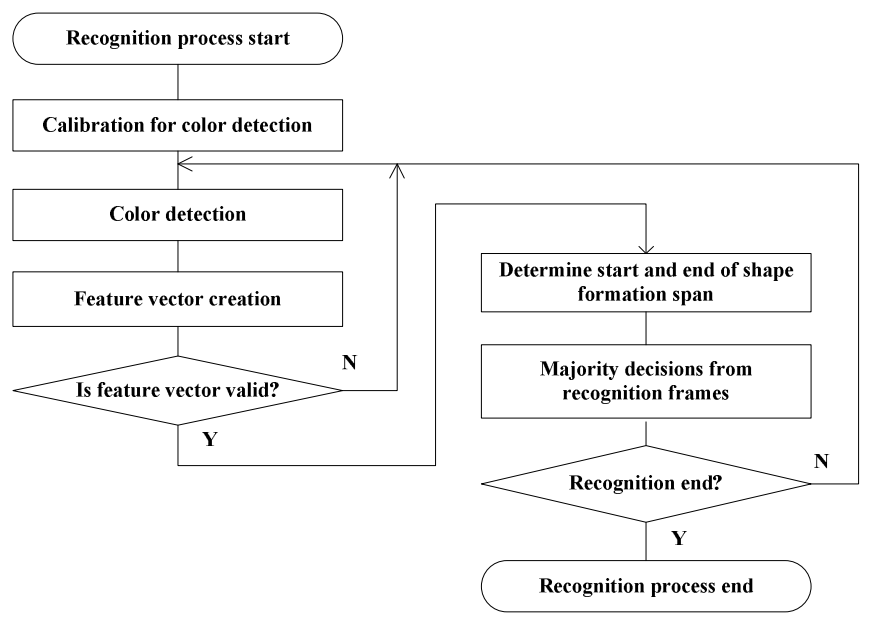

Fig. 7. Shape recognition sequence 


\section{Experiments and Evaluation}

Five illumination environments were used in this experiment. The experimental conditions are summarized in Table 4. The camera used in this experiment was the same as in section 3.2. In this experiment, three cases are considered in a nominal illumination environment of about 100 Lux to confirm the effect of a difference in background scenery in addition to the difference in brightness. The distance between hand and camera was set to about $50 \mathrm{~cm}$. Both right and left hand shapes were simultaneously evaluated to take practical usage into account. 15 typical hand shapes were formed sequentially, and this sequence was repeated 10 times, so the total number of test shapes was 150 .

Table 4. Experimental conditions for evaluating recognition performance

\begin{tabular}{|c|c|c|c|c|}
\hline & Illumination & Position of room light & Influence of sunlight & Background \\
\cline { 1 - 3 } Condition1 & \multirow{3}{*}{100 Lux } & Overhead & Small & White board \\
\cline { 1 - 3 } & Condition2 & Right slanting, Left slanting & Small & Bookshelf \\
\cline { 3 - 5 } Condition3 & All lights turned off & Medium & None \\
\hline Condition4 & 10 Lux & $\begin{array}{c}\text { Only overhead light turned on. All } \\
\text { others off }\end{array}$ & Small & Wall \\
\hline Condition5 & 400 Lux & All lights turned off & Large & None \\
\hline
\end{tabular}

Two methods were applied for this experiment and the results compared. In one method, only the one frame in the center of the recognition span was used, and in the other a majority decision was made, based on all the recognition results for recognition frames which were the latter half of the shape formation span. Table 5 and Table 6 show the experimental recognition results. The experiment demonstrated that use of a majority decision improved the recognition accuracy under low illumination conditions.

It has been verified that the proposed method of using color calibration by a clustering scheme and a voting method, based on a majority decision of the recognition results from each frames are effective in ensuring a high performance in hand shape recognition. The cause of the small number of failures $(2 \%)$ with majority voting method is due to the color detection error, that is, lack of color detection. These success ratios from $98 \%$ to $100 \%$ seem to be sufficient considering the application of intention transmission in daily life.

Table 5. Recognition experiment results without majority voting method

\begin{tabular}{|c|c|c|c|c|c|c|c|}
\hline & Number & \multicolumn{3}{|c|}{ Right hand } & \multicolumn{3}{c|}{ Left hand } \\
\cline { 3 - 8 } & Shapes & Success frames & Failure frames & Success ratio & Success frames & Failure frames & Success ratio \\
\hline Condition1 & 150 & 135 & 15 & $90.0 \%$ & 140 & 10 & $93.3 \%$ \\
\hline Condition2 & 150 & 142 & 8 & $94.7 \%$ & 143 & 7 & $95.3 \%$ \\
\hline Condition3 & 150 & 147 & 3 & $98.0 \%$ & 150 & 0 & $100.0 \%$ \\
\hline Condition4 & 150 & 132 & 18 & $88.0 \%$ & 137 & 13 & $91.3 \%$ \\
\hline Condition5 & 150 & 147 & 3 & $98.0 \%$ & 143 & 7 & $95.3 \%$ \\
\hline
\end{tabular}


Table 6. Recognition experiment results with majority voting method

\begin{tabular}{|c|c|c|c|c|c|c|c|}
\hline & Number & \multicolumn{3}{|c|}{ Right hand } & \multicolumn{3}{c|}{ Left hand } \\
\cline { 3 - 8 } & Shapes & Success frames & Failure frames & Success ratio & Success frames & Failure frames & Success ratio \\
\hline Condition1 & 150 & 147 & 3 & $98.0 \%$ & 149 & 1 & $99.3 \%$ \\
\hline Condition2 & 150 & 150 & 0 & $100.0 \%$ & 150 & 0 & $100.0 \%$ \\
\hline Condition3 & 150 & 150 & 0 & $100.0 \%$ & 150 & 0 & $100.0 \%$ \\
\hline Condition4 & 150 & 149 & 1 & $99.3 \%$ & 147 & 3 & $98.0 \%$ \\
\hline Condition5 & 150 & 150 & 0 & $100.0 \%$ & 150 & 0 & $100.0 \%$ \\
\hline
\end{tabular}

\section{Conclusion}

This paper presents a hand shape recognition method using colored gloves which takes into account the surrounding environment. Two methods are proposed to realize a high recognition performance under different environmental conditions. The hue value regions are decided based on a k-means clustering result, for accurate color detection. The duration or span of each hand shape formation is introduced and the use of a majority decision based on recognition results from recognition frames, which are those in the second half of the hand formation period, is proposed as a way to raise the recognition success ratio. Evaluation tests were carried out for different five cases. It was verified that the recognition success ratio for hand shape recognition can achieve $98 \%$ to $100 \%$ and the proposed methods are viable as means to improve recognition performance.

\section{References}

1. Khan, Z.R., Ibraheem, A.N.: Comparative Study of Hand Gesture Recognition System. In: Proc. of International Conference of Advanced Computer Science \& Information Technology in Computer Science \& Information Technology (CS \& IT), vol. 2(3), pp. 203-213 (2012)

2. Baatar, B., Tanaka, J.: Comparing Sensor Based and Vision Based Techniques for Dynamic Gesture Recognition. In: The 10th Asia Pacific Conference on Computer Human Interaction, APCHI 2012, Poster 2P-21 (2012)

3. Matsuda, Y., Sakuma, I., Jimbo, Y., Kobayashi, E., Arafune, T., Isomura, T.: Development of Finger Braille Recognition System. Journal of Biometrical Science and Engineering 5(1), 54-65 (2010)

4. Yoruk, E., Konukoglu, E., Sankur, B., Darbon, J.: Shape - Based Hand Recognition. IEEE Transactions on Image Processing 15(7), 1803-1815 (2006)

5. Suzuki, I., Nishimura, Y., Horiuchi, Y., Kuroiwa, S.: Sign Language Recognition from Video Using Particle Filter and Hidden Markov Model. IEICE Technical Report 110(384), 25-30 (2011) (Japanese)

6. Tanibata, N., Shimada, N., Shirai, Y.: Extraction of Hand Features for Recognition of Sign Language Words. In: Proc. of Int. Conference on Vision Interface, pp. 391-398 (2002)

7. Wang, Y.R., Popović, J.: Real-Time Hand-Tracking with a Color Glove. ACM Transactions on Graphics (TOG) 28(3), Article No. 63 (2009)

8. Sugaya, T., Suzuki, T., Nishimura, H., Tanaka, H.: Basic Investigation into Hand Shape Recognition Using Colored Gloves Taking Account of the Peripheral Environment. In: Yamamoto, S. (ed.) HIMI/HCII 2013, Part I. LNCS, vol. 8016, pp. 133-142. Springer, Heidelberg (2013) 